\title{
NT5DC2 is a novel prognostic marker in human hepatocellular carcinoma
}

\author{
JIEMIN CHEN $^{1}$, JIANZHONG CAO ${ }^{1}$, PENGHUI WANG ${ }^{2}$ and XIAODONG HE ${ }^{1}$ \\ ${ }^{1}$ Department of General Surgery, Peking Union Medical College Hospital, Peking Union Medical College and Chinese \\ Academy of Medical Sciences, Beijing 100730; \\ ${ }^{2}$ Department of General Surgery, Beijing Tiantan Hospital, Capital Medical University, Beijing 100070, P.R. China
}

Received January 30, 2020; Accepted July 1, 2020

DOI: $10.3892 / \mathrm{ol} .2020 .11931$

\begin{abstract}
Reliable biomarkers for the prognosis of hepatocellular carcinoma (HCC) are rare, and novel biomarkers are required for the appropriate management of $\mathrm{HCC}$. 5 '-Nucleotidase domain containing 2 (NT5DC2) acts as an oncogene in various tumors, but its functions as a biomarker have not been confirmed. Therefore, the present study aimed to resolve these functions by analyzing the prognostic value of NT5DC2 in patients with HCC. A tissue microarray (TMA) was prepared and NT5DC2 expression was measured via IHC staining in TMA dots. The liver cancer (LIHC) cohort in The Cancer Genome Atlas (TCGA) was enrolled as a secondary cohort. Kaplan-Meier survival analyses and Cox regression models were used for assessment of the prognostic value of $N T 5 D C 2$. Gene set enrichment analysis (GSEA) was performed in TCGA LIHC cohort. A total of 134 patients with $\mathrm{HCC}$ were retrospectively enrolled in the Peking Union Medical College Hospital cohort and clinical data were collected. A total of 359 patients with HCC in TCGA were enrolled as TCGA cohort. NT5DC2 was used as an indicator of overall survival (OS) and relapse-free survival (RFS) in multiple cohorts. In the multivariate Cox regression model, NT5DC2 upregulation was an independent prognostic factor of OS in both cohorts. GSEA indicated the enrichment of a series of survival- and metastasis-related gene-sets, such as LEE_LIVER_CANCER_SURVIVAL_UP and LIAO_ METASTASIS. Collectively, it was suggested that NT5DC 2 upregulation was associated with poor OS and RFS in HCC, and was a potential predictive marker for HCC stratification.
\end{abstract}

Correspondence to: Professor Xiaodong He, Department of General Surgery, Peking Union Medical College Hospital, Peking Union Medical College and Chinese Academy of Medical Sciences, 1 Shuaifuyuan, Wangfujing, Dongcheng, Beijing 100730, P.R. China E-mail: hexdpumch@sina.com

Key words: hepatocellular carcinoma, prognostic marker, 5 '-nucleotidase domain containing 2 , overall survival, cancer biomarker

\section{Introduction}

Hepatocellular carcinoma (HCC) is the most common human liver malignancy. Worldwide, the estimated number of mortalities caused by HCC is $\sim 810,000$ per year, while the estimated number of new cases of HCC is $\sim 854,000$ per year (1). As a nation with a large population and high prevalence of hepatitis B virus (HBV) and HCV, China accounts for $\sim 422,100 /$ year of total HCC mortalities worldwide (2). Thus, the mortality and severity of HCC represent an urgent health problem in China.

Current management of HCC includes potentially curative surgical resection and non-surgical therapeutics, such as radiofrequency ablation, percutaneous ethanol injection and trans-arterial embolization (3). Moreover, combined chemotherapy and recently developed immunotherapies constitute the treatment of patients with advanced HCC (3). However, it remains difficult to predict overall survival (OS) of patients with HCC for treatment selection. Tumor classification systems, such as TNM, Okuda, Cancer of the Liver Italian Program and Barcelona Clinic Liver Cancer, have been widely used for prognostic assessment $(4,5)$. However, due to the heterogeneity of malignancies, there is still no consensus on the most appropriate staging system for predicting the survival of patients with $\mathrm{HCC}(6,7)$. Previous studies have been conducted to identify potential biomarkers. It has been revealed that frequently used biomarkers, such as $\alpha$-fetoprotein (AFP) (8) and carbohydrate antigen 19-9 (CA19-9) (9), have a relatively poor performance as predictors of long-term outcome.

Recently, there have been studies on the mechanism of 5'-nucleotidase domain containing 2 (NT5DC2) in some malignancies, including gliomas (10) and liver cancer types (11). NT5DC2 has been reported to be associated with certain types of mental disorders, including schizophrenia, attention deficit hyperactivity disorder $(12,13)$ and borderline personality disorders (14). NT5DC2 promotes tumorigenesis of glioma stem-like cells by inducing the expression of FYN proto-oncogene (Fyn) (10), as well as increases tumor cell proliferation in $\mathrm{HCC}$ by stabilizing epidermal growth factor receptor (11). Currently, to the best of our knowledge, there is no research focusing on the value of NT5DC2 in the prognosis of $\mathrm{HCC}$. 
The present study aimed to investigate the suitability of NT5DC2 as a novel prognostic predictor for HCC.

\section{Materials and methods}

Data source. The latest liver cancer (LIHC) project data, including Level 3 RNA sequencing (RNA-seq) data and clinical data, were obtained using the $\mathrm{R}$ package, TCGAbiolinks v2.16.0 (15), as an independent validation cohort. There were 377 cases available including 255 males and 122 females. The age range was between 16 to 90 , with a median of 61. mRNA gene expression levels are presented as a normalized value of level 3 RNA-seq data. Then, NT5DC2 mRNA expression was compared between cancerous and paracancerous tissues. In HCC samples, patients were assigned into two equal groups (NT5DC2-High and NT5DC2-Low) based on the median normalized value of NT5DC2. The reciprocal The Cancer Genome Atlas (TCGA) clinical data (updated on 2019/08/08), comprising age, sex, weight, Child-Pugh score (16), TNM stage (17), residual tumor, Edmondson grade (18), relapse-free survival (RFS) and OS, were collected for further statistical analysis.

The Sequence Read Archive (SRA) dataset, SRP174991, including paired HCC sample RNA-seq raw data (19), were downloaded using the Linux package, prefetch (included in the Sra-tools version 2.10.6, https://github.com/ncbi/sra-tools).

Sample and clinical data collection. Patients diagnosed with HCC, receiving surgical resection between January 2008 and December 2015, in the Department of Hepatology and the Department of General Surgery, Peking Union Medical College Hospital (PUMCH), were retrospectively enrolled in this study. There were 134 cases available including 114 males and 20 females. The age range was between 32 to 82 years, with a median of 56 years. The reciprocal clinical data including age, sex, surface antigen of the hepatitis B virus (HBsAg), Anti-hepatitis C (HCV), aspartate transaminase, alanine aminotransferase (ALT), $\gamma$-glutamyl transpeptidase (GGT), alkaline phosphatase, AFP, CA19-9, TNM stage and Edmonson grade, were obtained via the Hospital Information System in PUMCH. The OS of enrolled patients was assessed via phone calls and routine clinical follow-up. This project was approved by the Ethic Committee, Peking Union Medical College Hospital (approval no. JS-1569) and had been performed in accordance with Declaration of Helsinki and its later amendments. Written informed consent was provided by all patients before surgery.

$R N A$ extraction and reverse transcription-quantitative $P C R$ $(R T-q P C R)$. Paired HCC samples, which were preserved frozen samples obtained during surgery, used in RT-qPCR were randomly chosen from the PUMCH cohort. Samples from patients with $\mathrm{HCC}$ were preserved in liquid nitrogen $\left(-196^{\circ} \mathrm{C}\right)$ immediately after resection.

Total RNA was extracted from liquid nitrogen-preserving tissues using TRIzol ${ }^{\circledR}$ reagent (Invitrogen; Thermo Fisher Scientific, Inc.) with Phasemaker ${ }^{\mathrm{TM}}$ tubes (Invitrogen; Thermo Fisher Scientific, Inc.), according to manufacturer's protocol. RT was performed using the GoScript ${ }^{\mathrm{TM}} \mathrm{RT}$ system
(Promega Corporation) with oligo-dT (15) primer at $42^{\circ} \mathrm{C}$ for $1 \mathrm{~h}$, according to the manufacturer's instructions. RT-qPCR was performed with 2X GoTaq Master Mix (cat. no. A6001; Promega Corporation) using an Applied Biosystems ${ }^{\mathrm{TM}} 7500$ Fast Dx Real-Time PCR System (Thermo Fisher Scientific, Inc.). Thermocycling conditions: $95^{\circ} \mathrm{C}$ for $2 \mathrm{~min}, 40$ cycles: $95^{\circ} \mathrm{C}$ for $10 \mathrm{sec}, 60^{\circ} \mathrm{C}$ for $30 \mathrm{sec}$. NT5DC2 mRNA expression was normalized to GAPDH mRNA expression. The gene expression was calculated via $2^{-\Delta \Delta C q}$ method (20). The primers validated for amplification efficiency and selected for RT-qPCR were as follows: NT5DC2 forward (F), 5'-GCAGCCATCTACGCC AACA-3' and reverse (R), 5'-TCACGGGCGGTACTGAAG A-3'; and GAPDH F, 5'-GGAGCGAGATCCCTCCAAAAT-3' and R, 5'-GGCTGTTGTCATACTTCTCATGG-3'.

Haemotoxylin-eosin $(H \& E)$ and immunohistochemical (IHC) staining of tissue microarrays (TMAs). TMAs were constructed from formalin-fixed, paraffin embedded tissue blocks stored at the Department of Pathology, PUMCH. All specimens were previously fixed in $10 \%$ neutral formalin solution at room temperature for $12 \mathrm{~h}$ before paraffin embedded. Tissue blocks of paired cancer, paracancerous tissue and randomly selected healthy tissue, as previously established by pathologists, were selected and TMAs were prepared. Tissue cores were re-embedded in paraffin, resected into $8-\mu \mathrm{m}$ thick slides. HE staining was performed using Gemini AS Automated Slide Stainer (Thermo Fisher Scientific, Inc.) with a normal H\&E staining program in room temperature. IHC staining was performed with HIER pH 6 retrieval. Slides were blocked by normal goat serum working solution (cat. no. SP-9001; OriGene Technologies, Inc.) at room temperature for $15 \mathrm{~min}$, then added $100 \mu \mathrm{l}$ Anti-NT5DC2 rabbit polyclonal antibody (1:50; cat. no. NBP2-13679; Novus Biologicals, LLC), and incubated at $37^{\circ} \mathrm{C}$ for $60 \mathrm{~min}$. Then, $100 \mu \mathrm{l}$ biotin-labeled goat anti-rabbit polyclonal antibody solution (ready to use; cat. no. SP-9001; OriGene Technologies, Inc.) was added to slides and incubated at room temperature for $15 \mathrm{~min}$. The reactions were visualized by diaminobenzidine (DAB) for $30 \mathrm{sec}$ at room temperature and counterstained with hematoxylin for $10 \mathrm{sec}$ at room temperature. Normal goat serum working solution, biotin-labeled goat anti-rabbit polyclonal antibody solution and streptavidin-HRP reagent were included in the SPlink Biotin-Streptavidin-horseradish peroxidase (HRP) Detection kit (cat. no. SP-9001; OriGene Technologies, Inc.). 1X DAB was diluted from the 20X DAB kit (cat. no. ZLI-9017; OriGene Technologies, Inc.). Sample results were evaluated by pathologists under a light microscope at the PUMCH. IHC-TMA slides were scanned using Panaroma SCAN II (3DHISTech Ltd.). In total, three random x 400 fields of view of the IHC chips were captured for mean optical density (MOD) analysis via ImageJ 1.52 (National Institutes of Health). The MOD was calculated as follows: MOD=Integrated OD/Sum area of samples. MODs were compared between cancerous tissues, para-HCC tissues and distal healthy liver tissues in IHC TMAs, and reciprocal receiver operating characteristic (ROC) curves were generated.

Statistical analysis. All experiments were repeated three times. $\mathrm{P}<0.05$ was considered to indicate a statistically significant 
difference. Quantitative analyses were performed using a Kolmogorov-Smirnov test for normality of the distribution and an F-test for equality of variances. Samples with normal distribution were represented as mean $\pm \mathrm{SD}$, while samples with non-normal distribution were represented as median (interquartile range). Categorical samples were expressed as $\mathrm{n} \%$ (n). Moreover, two groups with normal distribution were compared via Student's t-test; two paired groups with normal distribution were compared using paired t-test. Multiple groups with normal distribution were compared using one-way ANOVA test followed by Tukey's post hoc test. Groups with non-normal distribution were compared using Mann-Whitney U test. Fisher's exact test and $\chi^{2}$ test were used for comparison of categorical data. Kaplan-Meier survival analyses and Rényi tests were performed between NT5DC2-High and NT5DC2-Low groups. ROC curve was used to assess the diagnostic value of NT5DC2 in IHC TMAs. The median cut-off value was selected according to ROC curve.

Univariate Cox proportional hazards regression and multivariate Cox proportional hazards regression models were applied to analyze the impact of potentially confounding factors on OS. Potential confounding factors in both groups were evaluated via a univariate Cox regression model. Potentially significant factors $(\mathrm{P}<0.1)$ in the univariate Cox regression model were included in a multivariate Cox regression model. The hazard ratio (HR), 95\% CI and statistical significance are presented in the tables. All statistical analyses were performed via $\mathrm{R}$ software 3.5.3 (21).

Bioinformatic analyses. To investigate NT5DC2 function in patients with HCC, an integrative analysis of NT5DC2 was performed. Patients of the LIHC dataset were categorized into two groups according to NT5DC2 expression: NT5DC2-Low and NT5DC2-High. Gene Set Enrichment Analysis (GSEA) was performed using GSEA version 4.0.1 with MSigDB 7.0 (22,23). Gene sets with significant enrichment were listed, and RNA-seq data from the SRA dataset were mapped to the reference genome using hisat2 v2.1.0 (24). The reads were processed with featureCounts v1.6.0 (25) and DESeq2 v1.24.0 (26). The references consisted of the human reference genome (GRCh38) and the Ensembl annotated human transcriptome (GRCh38.v98). Normalized values were compared between the two groups and NT5DC2 expression levels were analyzed using the $\mathrm{R}$ package, ggplot2 v3.2.1 (https://ggplot2.tidyverse.org/). A Kaplan-Meier survival curve was generated using the R packages, survival v2.44-1.1 (https://CRAN.R-project.org/package=survival)and survminer v0.4.6 (https://rpkgs.datanovia.com/survminer/index.html).

\section{Results}

NT5DC2 mRNA is upregulated in cancerous tissue compared with paracancerous tissue in multiple datasets. In the LIHC dataset of TCGA, 377 cases were available, but eight cases were excluded due to the lack of RNA-seq data. Moreover, 10 cases of non-HCC liver cancer were excluded. One case was excluded due to the lack of OS data. Finally, 358 patients, for whom survival data were available, were included in the analysis. A total of $358 \mathrm{HCC}$ tissues and 40 para-HCC tissues were examined. Normalized NT5DC2 expression was significantly upregulated in $358 \mathrm{HCC}$ tissues compared with the para-HCC tissues $(\mathrm{P}<0.0001$; Fig. 1A). The available paired samples were selected $(n=50)$ in TCGA LIHC dataset, and a ROC curve analysis was performed. The area under the curve (AUC) of paired HCC samples was 0.942 (Fig. 1B).

In the SRP174991 dataset, NT5DC2 expression was compared in 35 pairs of HCC and para-HCC samples from patients with HBV-related HCC. The results demonstrated a significant upregulation of NT5DC2 in HCC tissues $(\mathrm{P}<0.0001$; Fig. 1C). In the 35 paired HCC SRP174991 samples, the AUC of paired HCC samples was 0.810 (Fig. 1D).

NT5DC2 mRNA expression was examined using RT-qPCR in $44 \mathrm{HCC}$ tissues and 44 para-HCC tissues randomly selected from the PUMCH cohort. The mRNA expression of NT5DC2 was significantly higher in cancerous compared with paracancerous tissues $(\mathrm{P}<0.05$; Fig. $1 \mathrm{E})$. ROC curve analysis was performed and the AUC was 0.637 (Fig. 1F).

IHC was conducted to analyze a TMA comprising 38 randomly selected non-HCC liver tissues, 32 paracancerous liver tissues and 32 HCC liver tissues (Fig. 1G). IHC staining identified a higher expression of NT5DC2 in HCC tissues compared with both para-HCC tissues $(\mathrm{P}<0.05)$ and distal healthy liver tissues $(\mathrm{P}<0.0001$; Fig. $1 \mathrm{H})$. Furthermore, ROC curves of NT5DC2 expression in HCC and para-HCC tissues were generated, and the AUC of NT5DC2 was 0.734 (Fig. 1I).

NT5DC2 gene upregulation reduces $O S$ and RFS in patients with HCC. In the LIHC cohort of TCGA, 358 patients with available survival data were included in the survival analysis. These patients were categorized into two groups based on the median value of NT5DC2 expression: NT5DC2-High $(\mathrm{n}=179)$ and NT5DC2-Low $(\mathrm{n}=179)$. The baseline data of the two groups are listed in Table I. Kaplan-Meier and Rényi analysis results demonstrated significant differences in $\mathrm{OS}(\mathrm{P}=0.030$; Fig. 2A) and RFS ( $\mathrm{P}=0.024$; Fig. 2B).

In the PUMCH cohort, a total of 134 patients were diagnosed with HCC and received surgical therapy (Table II). These patients were retrospectively enrolled in this study and categorized into two groups according to the median IHC MOD: NT5DC2-High $(\mathrm{n}=67)$ and NT5DC2-Low $(\mathrm{n}=67)$. Kaplan-Meier analysis was performed, and the Rényi test indicated that patients in the NT5DC2-High group had a significantly poorer OS $(\mathrm{P}=0.039)$ compared with the NT5DC2-Low group (Fig. 2C).

NT5DC2 gene upregulation is a risk factor associated with poor OS and RFS. In TCGA LIHC cohort, 358 patients with available survival data were categorized into two groups based on the median value of NT5DC2 expression: NT5DC2-High $(\mathrm{n}=179)$ and NT5DC2-Low $(\mathrm{n}=179)$. It was found that the two groups were different in age $(\mathrm{P}=0.013)$, weight $(\mathrm{P}=0.003)$, TNM stage $(\mathrm{P}<0.001)$ and Edmondson grade $(\mathrm{P}=0.026$; Table I).

To exclude potential confounding biases, clinically significant variables, such as age, sex, NT5DC2/1,000 units (NT5DC2/1,000), TNM stage and Edmondson grade were included in univariate Cox proportional hazards regression models to evaluate their influence on OS. The results suggested that both NT5DC2/1,000 $(\mathrm{P}=0.001 ; \mathrm{HR}=2.227$; 95\% $\mathrm{CI}=1.369-3.625)$ and $\mathrm{TNM}$ stage $(\mathrm{P}<0.001 ; \mathrm{HR}=1.512$; 
A

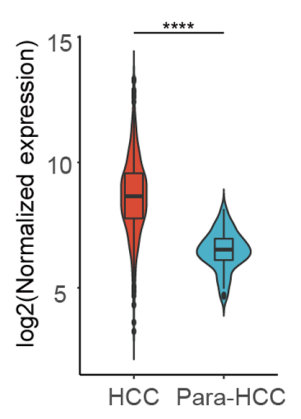

D NT5DC2 CA vs. PARA

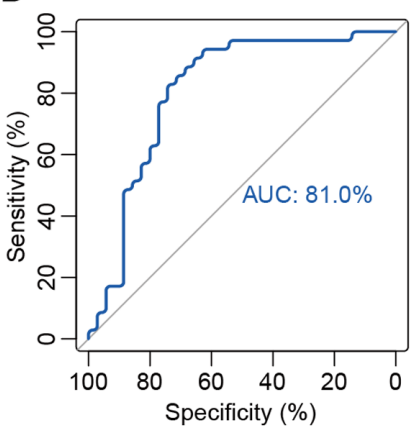

B

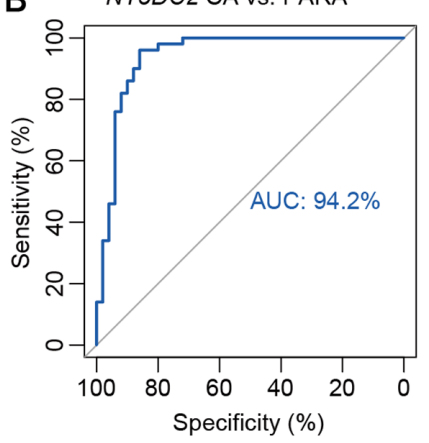

E

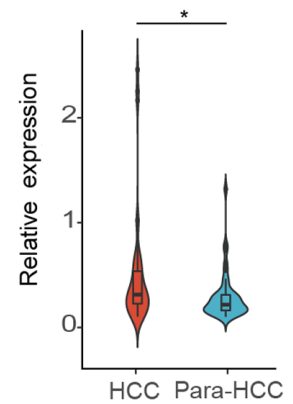

C

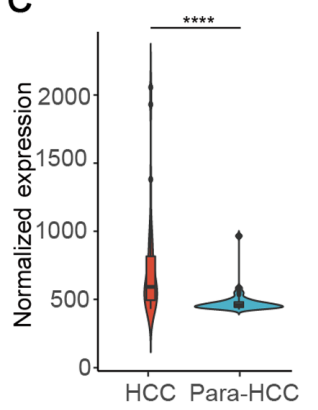

G

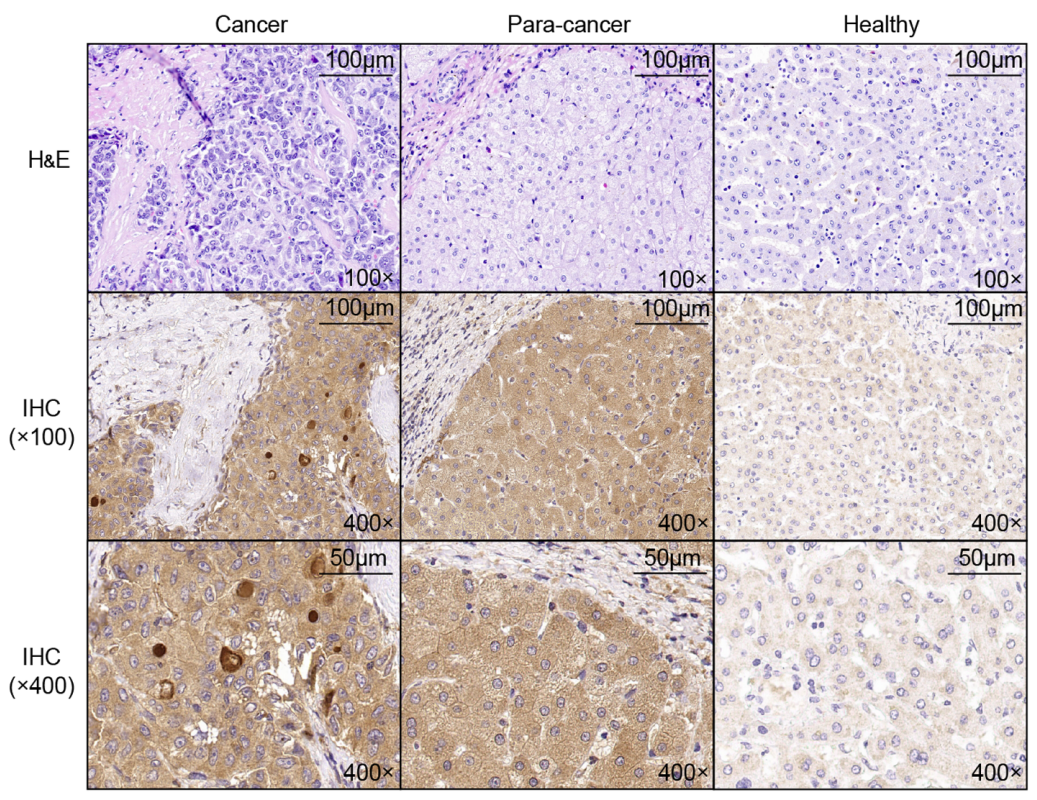

F NT5DC2 CA vs. PARA

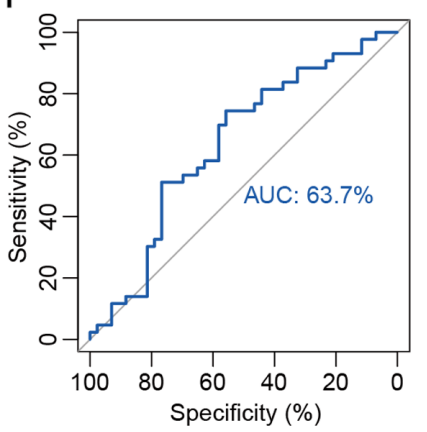

$\mathrm{H}$

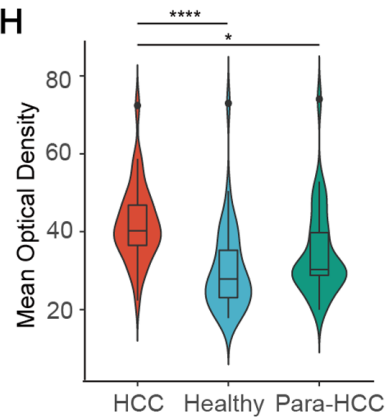

I

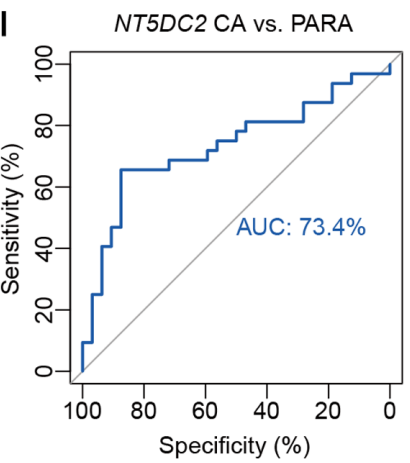

Figure 1. NT5DC2 is upregulated in cancerous tissue compared with paracancerous tissue in multiple datasets. (A) Violin plot of the difference in NT5DC2 expression value between HCC and paired para-HCC samples in TCGA LIHC cohort. (B) ROC curve of NT5DC2 expression in distinguishing between HCC and paired para-HCC tissues in TCGA LIHC cohort. The AUC was calculated and illustrated. (C) Violin plot of the difference in NT5DC2 expression value between HCC and paired para-HCC samples in SRP174991. (D) ROC curve of NT5DC2 expression for distinguishing between HCC and paired para-HCC tissues in SRP174991. AUC was calculated and illustrated. (E) Violin plot of the difference in NT5DC2 expression between HCC and paired para-HCC samples in selected HCC and paired para-HCC samples using reverse transcription-quantitative PCR. (F) ROC curve of NT5DC2 expression for distinguishing between HCC and paired para-HCC tissues in SRP174991. AUC was calculated and illustrated. (G) Selected tissue specimens in TMA of HCC, para-HCC and healthy liver samples stained using H\&E or IHC. (H) Violin plot demonstrating the difference in NT5DC2 expression between HCC and paired para-HCC samples in a TMA of the PUMCH cohort. Expression between groups were compared using one-way ANOVA followed by Tukey's test. (I) ROC curve of $N T 5 D C 2$ expression for distinguishing between HCC and paired para-HCC tissues in IHC-TMA of HCC, para-HCC and healthy liver samples. AUC was calculated and illustrated. " $\mathrm{P}<0.05,{ }^{* * * * *} \mathrm{P}<0.0001$. ROC, receiver operating characteristic; AUC, area under the curve; NT5DC2, 5'-nucleotidase domain containing 2; HCC, hepatocellular carcinoma; TCGA, The Cancer Genome Atlas; LIHC, liver cancer; TMA, tissue microarray; H\&E, hematoxylin and eosin; IHC, immunohistochemistry; PUMCH, Peking Union Medical College Hospital; CA, cancer; PARA, paracancerous.

95\% CI=1.284-1.781) were risk factors associated with reduced OS (Table II). These potential risk factors were included in a multivariate Cox proportional hazards regression model, which indicated that $N T 5 D C 2 / 1,000(\mathrm{P}=0.043 ; \mathrm{HR}=1.695$; 

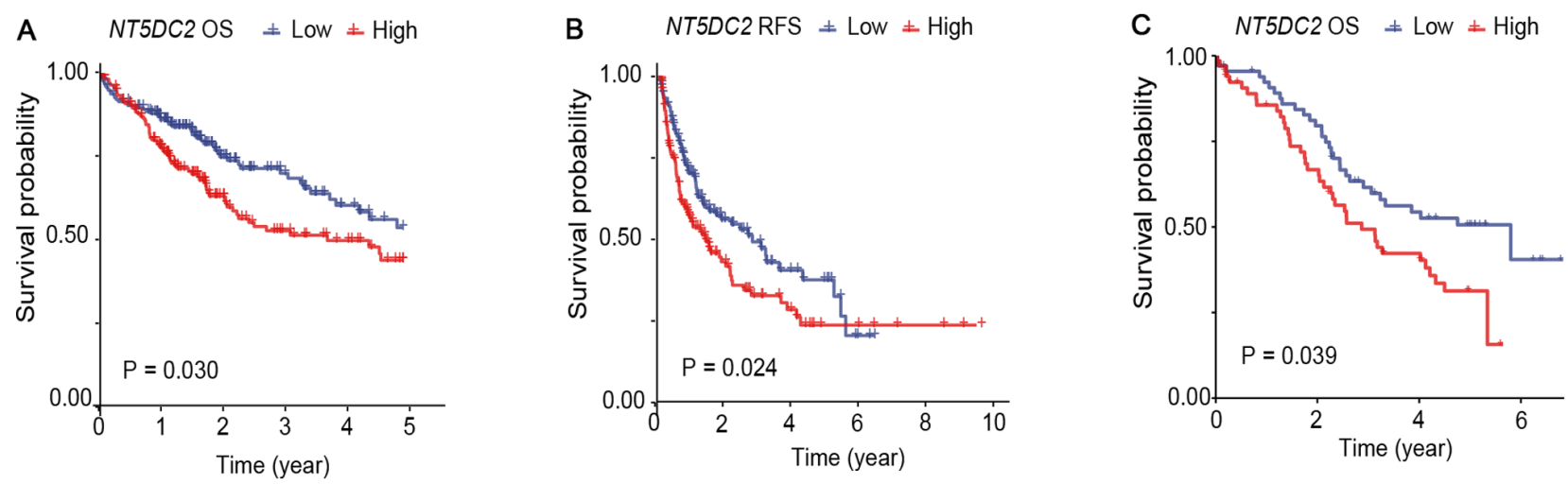

Figure 2. NT5DC2 gene upregulation reduces OS and RFS in patients with HCC. (A) Kaplan-Meier curve of time to all-cause mortality over 5 years in TCGA LIHC cohort. (B) Kaplan-Meier curve of time to tumor recurrence over 10 years in TCGA LIHC cohort. (C) Kaplan-Meier curve of time to all-cause mortality over 7 years in the PUMCH cohort. OS, overall survival; RFS, relapse-free survival; NT5DC2, 5'-nucleotidase domain containing 2; HCC, hepatocellular carcinoma; CA, cancer; PARA, paracancerous; TCGA, The Cancer Genome Atlas; LIHC, liver cancer; PUMCH, Peking Union Medical College Hospital.

Table I. Baseline characteristics of TCGA LIHC dataset $(n=358)$.

\begin{tabular}{|c|c|c|c|}
\hline Characteristic & NT5DC2-High & NT5DC2-Low & P-value \\
\hline Age, years & $57.78(13.95)$ & $61.26(12.33)$ & 0.013 \\
\hline Sex & & & 0.164 \\
\hline Male & $64.3(115)$ & $71.5(128)$ & \\
\hline Female & $35.7(64)$ & $28.5(51)$ & \\
\hline Weight, kg & $66.0(20.0)$ & $73.0(24.5)$ & 0.003 \\
\hline Child-Pugh score & & & 0.138 \\
\hline Grade A & $51.4(92)$ & $67.0(120)$ & \\
\hline Grade B & $7.3(13)$ & $4.4(8)$ & \\
\hline Grade C & $0(0)$ & $0.6(1)$ & \\
\hline TNM Stage & & & $<0.001$ \\
\hline Stage I & $35.8(64)$ & $57.2(103)$ & \\
\hline Stage II & $26.8(48)$ & $18.9(34)$ & \\
\hline Stage III & $30.2(54)$ & $15.6(28)$ & \\
\hline Stage IV & $1.1(2)$ & $1.1(2)$ & \\
\hline Residual tumor & & & 0.650 \\
\hline R0 & $88.3(158)$ & $88.3(159)$ & \\
\hline $\mathrm{R} 1$ & $3.4(6)$ & $4.5(8)$ & \\
\hline $\mathrm{R} 2$ & $0(0)$ & $0.6(1)$ & \\
\hline $\mathrm{Rx}$ & $6.7(12)$ & $4.5(8)$ & \\
\hline Edmondson grade & & & 0.026 \\
\hline G1 & $10.1(18)$ & $19.6(35)$ & \\
\hline G2 & $45.8(82)$ & 49.7 (89) & \\
\hline G3 & $38.0(68)$ & $28.5(51)$ & \\
\hline G4 & $3.9(7)$ & $2.2(4)$ & \\
\hline
\end{tabular}

Data are presented as the mean $\pm \mathrm{SD}$, median and interquartile range or $\mathrm{n} \%(\mathrm{n})$. NT5DC2-High, $\mathrm{n}=179$. NT5DC2-Low, $\mathrm{n}=179$. TCGA, The Cancer Genome Atlas.

95\% $\mathrm{CI}=1.016-1.727)$ and TNM stage $(\mathrm{P}<0.001 ; \mathrm{HR}=1.460$; $95 \% \mathrm{CI}=1.235-1.727)$ were independent risk factors for reduced OS (Table II).
Age, sex and TNM stage were also included in univariate Cox proportional hazards regression models to evaluate the impact of these factors on RFS. The results demonstrated that NT5DC2/1,000 $(\mathrm{P}<0.001 ; \mathrm{HR}=2.360 ; 95 \% \mathrm{CI}=1.547-3.599)$ and TNM stage $(\mathrm{P}<0.001 ; \mathrm{HR}=1.515 ; 95 \% \mathrm{CI}=1.312-1.750)$ were risk factors for shorter RFS (Table III). These potential risk factors were included in a multivariate Cox proportional hazards regression model, which identified that the NT5DC2/1,000 $(\mathrm{P}=0.011 ; \mathrm{HR}=1.780 ; 95 \% \mathrm{CI}=1.143-2.772)$ and TNM stage $(\mathrm{P}<0.001 ; \mathrm{HR}=1.444 ; 95 \% \mathrm{CI}=1.242-1.678)$ was independent risk factors for reduced RFS (Table III).

In the PUMCH dataset, multiple factors, including ALT, GGT and CA19-9, significantly differed between NT5DC2-High and NT5DC2-Low groups (Table IV). These potential risk factors, age, sex, NT5DC2-IHC MOD/10 units (NT5DC2/10), TNM stage, AFP per 1,000 units (AFP/1,000) and HBsAg were individually included in univariate Cox proportional hazards regression model. Univariate Cox regression models suggested that NT5DC2/10 ( $\mathrm{P}=0.002$; $\mathrm{HR}=1.185 ; 95 \% \mathrm{CI}=1.064-1.320), \mathrm{AFP} / 1,000 \quad(\mathrm{P}<0.001$; $\mathrm{HR}=1.017$; 95\% $\mathrm{CI}=1.007-1.026)$ and $\mathrm{TNM}$ stage $(\mathrm{P}<0.001$; $\mathrm{HR}=1.703$; $95 \% \mathrm{CI}=1.338-2.168)$ were risk factors for poor OS (Table V). Potential risk factors were included in a multivariate Cox proportional hazards regression model, indicating that NT5DC2/10 $(\mathrm{P}<0.001 ; \mathrm{HR}=1.219$; 95\% $\mathrm{CI}=1.090-1.364)$ and $\mathrm{TNM}$ stage $(\mathrm{P}<0.001 ; \mathrm{HR}=1.830$; 95\% $\mathrm{CI}=1.409-2.377)$ were independent risk factors for poor OS (Table V).

NT5DC2 may participate in cancer-related biological processes in HCC. GSEA was performed in TCGA LIHC cohort. Gene expression data of TCGA LIHC cohort were divided into two groups based on normalized NT5DC2 gene expression: NT5DC2-High and NT5DC2-Low. GSEA was performed based on the phenotype of the group (Fig. 3B). In cancer-related phenotype, a liver cancer survival related gene set, LEE_LIVER_CANCER_SURVIVAL_UP, and a metastasis-related gene set, LIAO_METASTASIS, were enriched. Moreover, a proliferation-related gene set was enriched, CHIANG_LIVER_CANCER_SUBCLASS PROLIFERATION_UP. It was suggested that the bile acid 
Table II. Cox Proportional Hazards regression models for overall survival in The Cancer Genome Atlas liver cancer cohort $(n=359)$.

Univariate

\begin{tabular}{|c|c|c|c|c|}
\hline \multirow[b]{2}{*}{ Variables } & & \\
\hline & HR $(95 \% \mathrm{CI})$ & P-value & HR $(95 \% \mathrm{CI})$ & $\mathrm{P}$-value \\
\hline Age, years & $1.012(0.998-1.026)$ & 0.109 & & \\
\hline Male & $0.841(0.585-1.210)$ & 0.351 & & \\
\hline$N T 5 D C 2 / 1,000$ & $2.227(1.369-3.625)$ & 0.001 & $1.695(1.016-1.727)$ & 0.043 \\
\hline TNM stage & $1.512(1.284-1.781)$ & $<0.001$ & $1.460(1.235-1.727)$ & $<0.001$ \\
\hline Edmondson grade & $1.081(0.852-1.372)$ & 0.522 & & \\
\hline
\end{tabular}

NT5DC2/1,000, NT5DC2 per 1,000 units; HR, hazard ratio.
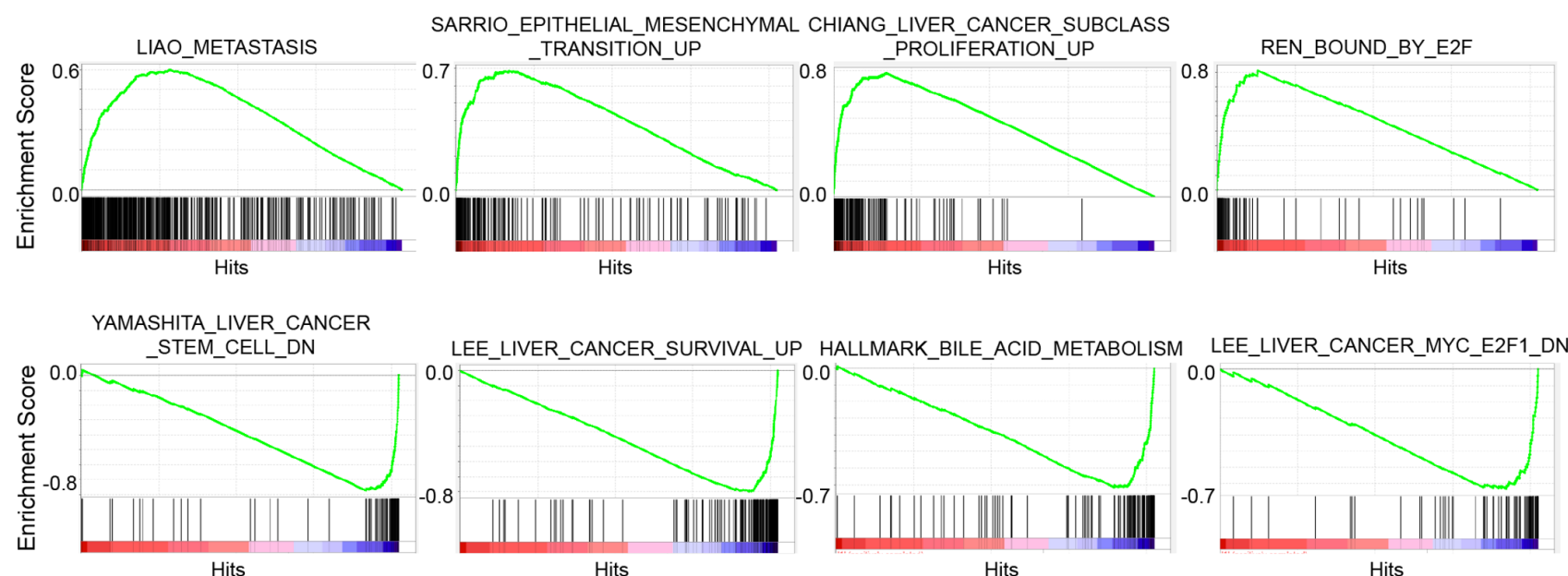

Hits
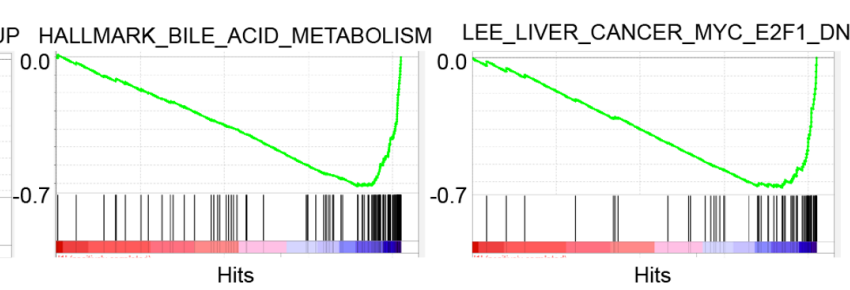

Figure 3. NT5DC2 may participate in cancer-related biological processes in HCC. Gene Set Enrichment Analysis demonstrated the enrichment score of multiple cancer-related pathways in NT5DC2-overexpressing patients in The Cancer Genome Atlas liver cancer cohort. In the bottom, the left side of this list (red color) contains genes upregulated in the gene set. The right side of the list (blue color) contains downregulated genes in the gene set. HCC, hepatocellular carcinoma.

may be downregulated, since the bile acid metabolism-related gene set was also enriched, HALLMARK_BILE_ACID_ METABOLISM.

Mechanically, two Cyclin-Rb-E2F pathway related gene sets, REN_BOUND_BY_E2F and LEE_LIVER_ CANCER_MYC_E2F1_DN, were significantly enriched. In addition, SARRIO_EPITHELIAL_ MESENCHYMAL_TRANSITION_UP, as an epithelial-mesenchymal-transition (EMT)-related gene set, was upregulated. Gene set YAMASHITA_LIVER_CANCER_ STEM_CELL_DN, which was related to liver cancer stem-like cells, was also enriched. The plot of GSEA enrichment is presented in Fig. 3.

\section{Discussion}

Reliable biomarkers may aid in the correct clinical decisions by surgeons when in dilemma over diagnosis (27). Most traditional markers of $\mathrm{HCC}$ are suitable for early diagnosis. However, commonly used biomarkers, such as AFP (28), carcinoembryonic antigen (29) and CA19-9 (30), have shown poor performance in HCC prognosis before surgery. The present study demonstrated that the evaluation of NT5DC2 expression via IHC staining and RNA-seq, but not RT-qPCR, was capable of distinguishing HCC tissues from paired para-HCC tissues. In the current study, RT-qPCR exhibited the lowest AUC, suggesting its unsuitability for clinical use. RNA-seq of HCC and para-HCC tissues resulted in a higher AUC compared with IHC staining. However, IHC staining is cheaper and simpler compared with RNA-seq, and the analysis is easily performed, especially in bioptic samples (31).

The present results suggested that NT5DC2 was a potential predictor of OS, although in TCGA LIHC cohort, the Rényi P-value was initially not significant. Notably, in this cohort, OS referred to all-cause mortality. It was identified that, in this cohort, the average age of enrolled patients at diagnosis was $\sim 60$ years. Therefore, long-term follow-up could exceed the life expectancy of enrolled patients. HCC has a comparatively low 5-year survival rate (32); therefore, an endpoint of follow-up was set up as the 5th year. Then, the Rényi P-value became significant. In the multivariate Cox regression model, $N T 5 D C 2$ was an independent risk factor for reduced OS and RFS, indicating that it was a potential prognostic factor for HCC. This Cox regression model also demonstrated that the TNM stage was associated with both OS and RFS, in accordance with previous studies (33-35). 
Table III. Cox Proportional Hazards regression model for relapse-free survival in The Cancer Genome Atlas liver cancer cohort $(n=359)$.

\begin{tabular}{|c|c|c|c|c|}
\hline \multirow[b]{2}{*}{ Variables } & \multicolumn{2}{|c|}{ Univariate } & \multicolumn{2}{|c|}{ Multivariate } \\
\hline & $\mathrm{HR}(95 \% \mathrm{CI})$ & P-value & $\mathrm{HR}(95 \% \mathrm{CI})$ & P-value \\
\hline Age, years & $0.996(0.984-1.008)$ & 0.481 & & \\
\hline Male & $1.056(0.761-1.466)$ & 0.744 & & \\
\hline$N T 5 D C 2 / 1000$ & $2.360(1.547-3.599)$ & $<0.001$ & $1.780(1.143-2.772)$ & 0.011 \\
\hline TNM stage & $1.515(1.312-1.750)$ & $<0.001$ & $1.444(1.242-1.678)$ & $<0.001$ \\
\hline Edmondson grade & $1.096(0.892-1.348)$ & 0.382 & & \\
\hline
\end{tabular}

NT5DC2/1,000, NT5DC2 per 1,000 units; HR, hazard ratio.

Table IV. Baseline data of PUMCH dataset $(n=134)$.

\begin{tabular}{|c|c|c|c|}
\hline Characteristic & NT5DC2-High & NT5DC2-Low & P-value \\
\hline $\mathrm{Age}^{\mathrm{a}}$, years & $55.27 \pm 11.44$ & $57.93 \pm 10.52$ & 0.164 \\
\hline Sex & & & 0.084 \\
\hline Male & $86.6(58)$ & $73.1(49)$ & \\
\hline Female & $13.4(9)$ & $26.9(18)$ & \\
\hline HBsAg, $\%$ positive & $79.1(53)$ & $73.1(49)$ & 0.544 \\
\hline Anti-HCV, $\%$ positive & $9.0(6)$ & $9.0(6)$ & 1.000 \\
\hline AST, U/1 & $37.0(37.5)$ & $36.00(25.5)$ & 0.140 \\
\hline ALT, U/1 & $39.0(36.0)$ & $29.00(29.2)$ & 0.010 \\
\hline GGT, U/1 & $69.0(98.5)$ & $55.00(59.5)$ & 0.015 \\
\hline ALP, U/1 & $76.0(43.0)$ & $78.00(49.5)$ & 0.478 \\
\hline AFP, ng/ml & $176.5(2019.0)$ & $30.00(481.9)$ & 0.063 \\
\hline $\mathrm{CA} 19-9, \mathrm{U} / \mathrm{ml}$ & $24.2(40.85)$ & $22.00(23.65)$ & $<0.001$ \\
\hline Edmondson grade & & & 0.777 \\
\hline G1 & $23.9(17)$ & $26.8(18)$ & \\
\hline $\mathrm{G} 2$ & $62.7(42)$ & $61.2(41)$ & \\
\hline G3 & $27.2(6)$ & $7.5(5)$ & \\
\hline G4 & $4.3(1)$ & $0(0)$ & \\
\hline TNM Stage & & & 0.732 \\
\hline $\mathrm{I}$ & $59.7(40)$ & $55.2(37)$ & \\
\hline II & $13.4(9)$ & $11.9(8)$ & \\
\hline III & $25.4(17)$ & $28.4(19)$ & \\
\hline IV & $1.5(1)$ & $4.5(3)$ & \\
\hline
\end{tabular}

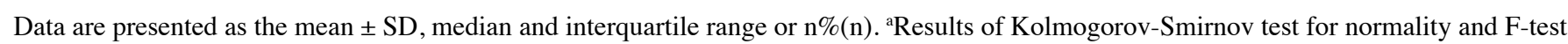
for equality of two variances $\mathrm{P}>0.05$. NT5DC2-High, $\mathrm{n}=67$. NT5DC2-Low, $\mathrm{n}=67$.

In the current study it was identified that whether IHC or RNA-sequencing were used, NT5DC2 could be a beneficial indicator of the prognosis of HCC. TNM stage and NT5DC2 were found to be independent risk factors; hence, $N T 5 D C 2$ can increase the prognostic value of the TNM stage. As a common method of pathological diagnosis, the accessibility of IHC is not inferior to serum biomarkers such as AFP (27). However, the present results indicated that AFP was a risk factor dependent on TNM stage. A previous study has shown that the increase in serum AFP levels was related to the tumor volume of HCC (36). Since TNM stage already contains the tumor volume, the prognostic value of AFP may be limited, especially when used simultaneously with the TNM stage (37). Therefore, NT5DC2 is more suitable as a supplement to TNM stage compared with AFP.

In the present study, GSEA analysis in NT5DC2-High and $N T 5 D C 2$-Low groups, and it was found that NT5DC 2 upregulation was associated with metastasis and survival, which was in accordance with the Kaplan-Meier analyses results. While the detailed mechanism of how NT5DC2 upregulation affects OS and RFS in patients with HCC remains elusive, the GSEA result 
Table V. Cox Proportional Hazards regression model of overall survival in the Peking Union Medical College Hospital cohort $(\mathrm{n}=134)$.

\begin{tabular}{llrrr}
\hline & \multicolumn{2}{c}{ Univariate } & & Multivariate \\
\cline { 2 - 3 } Variables & HR $(95 \% \mathrm{CI})$ & P-value & HR $(95 \%$ CI $)$ & \\
\hline Age, years & $0.995(0.973-1.019)$ & 0.710 & & \\
Male & $1.221(0.625-2.384)$ & 0.559 & $1.219(1.090-1.364)$ & $<0.001$ \\
NT5DC2/10 & $1.185(1.064-1.320)$ & $<0.001$ & $1.008(0.998-1.018)$ & 0.136 \\
AFP/1000 & $1.017(1.007-1.026)$ & $<0.001$ & $1.830(1.409-2.377)$ & $<0.001$ \\
TNM stage & $1.703(1.338-2.168)$ & 0.433 &
\end{tabular}

NT5DC2/10, NT5DC2 per 10 units; AFP/1,000, AFP per 1,000 units; HBsAg, surface antigen of the hepatitis B virus.

may provide some suggestions. For example, the enriched gene set included REN_BOUND_BY_E2F, a gene set of E2F bound targets, which was a result of Anti-E2F chromatin immunoprecipitation and sequencing in mammal cell-lines (38). Thus, NT5DC2 upregulation could reflect Cyclin-retinoblastoma tumor suppressor (Rb)-E2F pathway activation (39). Elevated E2F or E2F target expression is associated with poor HCC prognosis $(40,41)$. In a previous study of HCC, overexpression of NT5DC2 significantly promoted the change of cell cycle from $G_{1}$ to $S$ phase and the expression of cyclins was upregulated (11). The Rb-E2F pathway is an important $G_{1} / S$ phase checkpoint, and the $\mathrm{G}_{1} / \mathrm{S}$ ratio change that occurs during NT5DC2 overexpression is likely due to the Cyclin-Rb-E2F pathway, but this requires further verification (39).

An enrichment in EMT-related genes was also identified in the current study, which was consistent with a previous report (42). EMT is a strong clinical indicator for HCC, and is crucial for HCC metastasis (42). It has been shown that transforming growth factor- $\beta$-induced EMT may also induce a stemness phenotype in HCC cell-lines (43). In glioma stem-like cells, NT5DC2 was revealed to be highly expressed and induce the upregulation of Fyn, resulting in an aggressive phenotype (10). Moreover, in the current study, the cancer stem cell-related dataset, YAMASHITA_LIVER_CANCER_ STEM_CELL_DN_96, was enriched in association with $N T 5 D C 2$ expression, as assessed using GSEA. However, the molecular mechanism via which NT5DC2 influences HCC stemness requires further investigation.

A strength of the current study was that the role of NT5DC2 as a risk factor for OS was demonstrated in two independent HCC cohorts. However, this study has some limitations. First, in the PUMCH cohort, $>70 \%$ of patients with HCC were HBVor HCV-positive. Thus, the results of IHC-stained TMA may not apply to patients from Western countries, where HBV/HCV infection rates are much lower (44). Second, this study did not demonstrate $N T 5 D C 2$ suitability as a serum biomarker, possibly representing a limitation to its clinical use. However, since previous IHC analysis of multiple biomarkers, including human epidermal growth factor receptor $2 \mathrm{~F}$ (45) and programmed death-ligand 1 (46), was highly consistent between surgical resection samples and bioptic samples, IHC staining may still be a suitable method for the analysis of bioptic samples.
Overall, the present study demonstrated the value of NT5DC2 as an prognostic biomarker in multiple HCC cohorts. In the multivariate Cox regression model, NT5DC2 upregulation was an independent risk factor of poor OS in both cohorts and poor RFS in LIHC cohort. GSEA indicated the enrichment of a series of survival- and metastasis-related gene-sets, such as LEE_LIVER_CANCER_SURVIVAL_UP and LIAO_METASTASIS.

\section{Acknowledgements}

Not applicable.

\section{Funding}

This study was supported by the National Natural Science Foundation of China (grant no. 81372578), Chinese Academy of Medical Sciences Innovation Fund (grant no. 2017-I2M-4-003).

\section{Availability of data and materials}

The datasets used or analyzed during the present study are available from the corresponding author upon reasonable request. The bioinformatics datasets generated and/or analyzed during the current study are available in The Cancer Genome Atlas repository, https://www.cancer.gov/tcga.

\section{Authors' contributions}

JMC and XDH was responsible for research design and provided study material. JMC provided the pathological sections and performed the immunochemical scoring together with JZC. PHW collected and assembled the clinical and follow-up data. JMC, JZC and PHW analyzed and interpreted the data. JMC and XDH drafted and finalized the manuscript. All authors read and approved the final version of the manuscript.

\section{Ethics approval and consent to participate}

This project was approved by the Ethic Committee, Peking Union Medical College Hospital (approval no. JS-1569) and had been performed in accordance with Declaration of 
Helsinki and its later amendments. All persons gave their written informed consent prior to their inclusion in the study. Any details that may disclose the identity of the subjects under study were not included in this study.

\section{Patient consent for publication}

Not applicable.

\section{Competing interests}

The authors declare that they have no competing interests.

\section{References}

1. Global Burden of Disease Cancer Collaboration, Fitzmaurice $\mathrm{C}$, Allen C, Barber RM, Barregard L, Bhutta ZA, Brenner H, Dicker DJ, Chimed-Orchir O, Dandona R, et al: Global, regional, and national cancer incidence, mortality, years of life lost, years lived with disability, and disability-adjusted life-years for 32 cancer groups, 1990 to 2015: A systematic analysis for the global burden of disease study. JAMA Oncol 3: 524-548, 2017.

2. Chen W, Zheng R, Baade PD, Zhang S, Zeng H, Bray F, Jemal A $\mathrm{Yu}$ XQ and He J: Cancer statistics in China, 2015. CA Cancer J Clin 66: 115-132, 2016.

3. NCCN: NCCN Clinical Practice Guidelines in Oncology (NCCN Guidelines $^{\circledR}$ ): Hepatobiliary Cancers (Version 3.2018), 2018.

4. Zhang JF, Shu ZJ, Xie CY, Li Q, Jin XH, Gu W, Jiang FJ and Ling CQ: Prognosis of unresectable hepatocellular carcinoma: Comparison of seven staging systems (TNM, Okuda, BCLC, CLIP, CUPI, JIS, CIS) in a Chinese cohort. PLoS One 9: e88182, 2014.

5. Chen ZH, Hong YF, Lin J, Li X, Wu DH, Wen JY, Chen J, Ruan DY, Lin Q, Dong M, et al: Validation and ranking of seven staging systems of hepatocellular carcinoma. Oncol Lett 14 705-714, 2017

6. Liu PH, Hsu CY, Hsia CY, Lee YH, Su CW, Huang YH, Lee FY, Lin HC and Huo TI: Prognosis of hepatocellular carcinoma: Assessment of eleven staging systems. J Hepatol 64: 601-608, 2016.

7. Sherman M: Staging for hepatocellular carcinoma: Complex and confusing. Gastroenterology 146: 1599-1602, 2014.

8. Ahmed Mohammed HF and Roberts LR: Should AFP (or any biomarkers) be used for HCC surveillance? Curr Hepatol Rep 16: $137-145,2017$.

9. Zhou L, Rui JA, Wang SB, Chen SG and Qu Q: Carbohydrate antigen 19-9 increases the predictive efficiency of $\alpha$-fetoprotein for prognosis of resected hepatocellular carcinoma. Am Surg 84: 80-85, 2018

10. Guo S, Ran H, Xiao D, Huang H, Mi L, Wang X, Chen L, Li D Zhang S, Han Q, et al: NT5DC2 promotes tumorigenicity of glioma stem-like cells by upregulating fyn. Cancer Lett 454 98-107, 2019.

11. Li KS, Zhu XD, Liu HD, Zhang SZ, Li XL, Xiao N, Liu XF Xu B, Lei M, Zhang YY, et al: NT5DC2 promotes tumor cell proliferation by stabilizing EGFR in hepatocellular carcinoma. Cell Death Dis 11: 335, 2020.

12. van Hulzen KJE, Scholz CJ, Franke B, Ripke S, Klein M, McQuillin A, Sonuga-Barke EJ; PGC ADHD Working Group, Kelsoe JR, Landén M, et al: Genetic overlap between attention-deficit/hyperactivity disorder and bipolar disorder: evidence from genome-wide association study meta-analysis. Biol Psychiatry 82: 634-641, 2017.

13. Zayats T, Jacobsen KK, Kleppe R, Jacob CP, Kittel-Schneider S, Ribasés M, Ramos-Quiroga JA, Richarte V, Casas M, Mota NR, et al: Exome chip analyses in adult attention deficit hyperactivity disorder. Transl Psychiatry 6: e923, 2016.

14. Prados J, Stenz L, Courtet P, Prada P, Nicastro R, Adouan W, Guillaume S, Olié E, Aubry JM, Dayer A and Perroud N: Borderline personality disorder and childhood maltreatment: A genome-wide methylation analysis. Genes Brain Behav 14: 177-188, 2015.

15. Colaprico A, Silva TC, Olsen C, Garofano L, Cava C, Garolini D, Sabedot TS, Malta TM, Pagnotta SM, Castiglioni I, et al: TCGAbiolinks: An R/Bioconductor package for integrative analysis of TCGA data. Nucleic Acids Res 44: e71, 2016.
16. Forner A, Reig $M$ and Bruix J: Hepatocellular carcinoma. Lancet 391: 1301-1314, 2018.

17. Amin M, Edge S, Greene FL, Schilsky RL, Byrd DR, Gaspar LE, Washington MK, Gershenwald JE, Compton CC and Hess KR (eds): AJCC cancer staging manual. 8th edition. Springer, New York, NY, 2017.

18. Zhou L, Rui JA, Zhou WX, Wang SB, Chen SG and Qu Q: Edmondson-Steiner grade: A crucial predictor of recurrence and survival in hepatocellular carcinoma without microvascular invasio. Pathol Res Pract 213: 824-830, 2017.

19. Jiang Y, Sun A, Zhao Y, Ying W, Sun H, Yang X, Xing B, Sun W, Ren L, Hu B, et al: Proteomics identifies new therapeutic targets of early-stage hepatocellular carcinoma. Nature 567: 257-261, 2019.

20. Livak KJ and Schmittgen TD: Analysis of relative gene expression data using real-time quantitative PCR and the 2(-Delta Delta C(T)) method. Methods 25: 402-408, 2001.

21. R Core Team: R: A language and environment for statistical computing. R Foundation for Statistical Computing, Vienna, Austria, 2018

22. Mootha VK, Lindgren CM, Eriksson KF, Subramanian A Sihag S, Lehar J, Puigserver P, Carlsson E, Ridderstråle M, Laurila E, et al: PGC-1alpha-responsive genes involved in oxidative phosphorylation are coordinately downregulated in human diabetes. Nat Genet 34: 267-273, 2003.

23. Subramanian A, Tamayo P, Mootha VK, Mukherjee S, Ebert BL, Gillette MA, Paulovich A, Pomeroy SL, Golub TR, Lander ES and Mesirov JP: Gene set enrichment analysis: A knowledge-based approach for interpreting genome-wide expression profiles. Proc Natl Acad Sci USA 102: 15545-15550, 2005.

24. Kim D, Langmead B and Salzberg SL: HISAT: A fast spliced aligner with low memory requirements. Nat Methods 12: 357-360, 2015.

25. Liao Y, Smyth GK and Shi W: featureCounts: An efficient general purpose program for assigning sequence reads to genomic features. Bioinformatics 30: 923-930, 2014.

26. Love MI, Huber W and Anders S: Moderated estimation of fold change and dispersion for RNA-seq data with DESeq2. Genome Biol 15: 550, 2014

27. Sia D, Villanueva A, Friedman SL and Llovet JM: Liver cancer cell of origin, molecular class, and effects on patient prognosis. Gastroenterology 152: 745-761, 2017.

28. Yang SL, Liu LP, Yang S, Liu L, Ren JW, Fang X, Chen GG and Lai PB: Preoperative serum $\alpha$-fetoprotein and prognosis after hepatectomy for hepatocellular carcinoma. Br J Surg 103: 716-724, 2016.

29. Liu J, Xia Y, Shi L, Li X, Wu L and Yan Z: Elevated serum carcinoembryonic antigen is associated with a worse survival outcome of patients after liver resection for hepatocellular carcinoma: A propensity score matching analysis. J Gastrointest Surg 20: 2063-2073, 2016

30. Hsu CC, Goyal A, Iuga A, Krishnamoorthy S, Lee V, Verna EC, Wang S, Chen FN, Rodriguez R, Emond J, et al: Elevated CA19-9 is associated with increased mortality in a prospective cohort of hepatocellular carcinoma patients. Clin Transl Gastroenterol 6: e74, 2015.

31. Suryavanshi M, Mehta A, Jaipuria J, Kumar D, Vishwakarma G, Panigrahi MK, Verma H, Saifi M, Sharma S, Tandon S, et al: Clinical utility of RT-PCR in assessing HER 2 gene expression versus traditional IHC and FISH in breast cancer patients. Breast Cancer 25: 416-430, 2018.

32. Zeng H, Zheng R, Guo Y, Zhang S, Zou X, Wang N, Zhang L, Tang J, Chen J, Wei K, et al: Cancer survival in China, 2003-2005: A population-based study. Int J Cancer 136: 1921-1930, 2015.

33. Tabrizian P, Jibara G, Shrager B, Schwartz M and Roayaie S: Recurrence of hepatocellular cancer after resection: Patterns, treatments, and prognosis. Ann Surg 261: 947-955, 2015.

34. Ma X, Gu J, Wang K, Zhang X, Bai J, Zhang J, Liu C, Qiu Q and Qu K: Identification of a molecular subtyping system associated with the prognosis of Asian hepatocellular carcinoma patients receiving liver resection. Sci Rep 9: 7073, 2019.

35. Cao J, Wang P, Chen J and He X: PIGU overexpression adds value to TNM staging in the prognostic stratification of patients with hepatocellular carcinoma. Hum Pathol 83: 90-99, 2019.

36. Liu C, Xiao GQ, Yan LN, Li B, Jiang L, Wen TF, Wang WT, $\mathrm{Xu}$ MQ and Yang JY: Value of $\alpha$-fetoprotein in association with clinicopathological features of hepatocellular carcinoma. World J Gastroenterol 19: 1811-1819, 2013.

37. Kamarajah SK, Frankel TL, Sonnenday C, Cho CS and Nathan $\mathrm{H}$ : Critical evaluation of the American joint commission on cancer (AJCC) 8th edition staging system for patients with hepatocellular carcinoma (HCC): A surveillance, epidemiology, end results (SEER) analysis. J Surg Oncol 117: 644-650, 2018. 
38. Ren B, Cam H, Takahashi Y, Volkert T, Terragni J, Young RA and Dynlacht BD: E2F integrates cell cycle progression with DNA repair, replication, and G(2)/M checkpoints. Genes Dev 16: 245-256, 2002.

39. Kent LN and Leone G: The broken cycle: E2F dysfunction in cancer. Nat Rev Cancer 19: 326-338, 2019.

40. Kent LN, Rakijas JB, Pandit SK, Westendorp B, Chen HZ, Huntington JT, Tang X, Bae S, Srivastava A, Senapati S, et al: E2f8 mediates tumor suppression in postnatal liver development. J Clin Invest 126: 2955-2969, 2016.

41. Kent LN, Bae S, Tsai SY, Tang X, Srivastava A, Koivisto C, Martin CK, Ridolfi E, Miller GC, Zorko SM, et al: Dosage-dependent copy number gains in E2f1 and E2f3 drive hepatocellular carcinoma. J Clin Invest 127: 830-842, 2017.

42. Giannelli G, Koudelkova P, Dituri F and Mikulits W: Role of epithelial to mesenchymal transition in hepatocellular carcinoma. J Hepatol 65: 798-808, 2016.
43. Malfettone A, Soukupova J, Bertran E, Crosas-Molist E, Lastra R, Fernando J, Koudelkova P, Rani B, Fabra Á, Serrano T, et al: Transforming growth factor- $\beta$-induced plasticity causes a migratory stemness phenotype in hepatocellular carcinoma. Cancer Lett 392: 39-50, 2017.

44. de Martel C, Maucort-Boulch D, Plummer M and Franceschi S: World-wide relative contribution of hepatitis $\mathrm{B}$ and $\mathrm{C}$ viruses in hepatocellular carcinoma. Hepatology 62: 1190-1200, 2015.

45. Wang T, Hsieh ET, Henry P, Hanna W, Streutker CJ and Grin A: Matched biopsy and resection specimens of gastric and gastroesophageal adenocarcinoma show high concordance in HER2 status. Hum Pathol 45: 970-975, 2014.

46. Wang H, Agulnik J, Kasymjanova G, Wang A, Jiménez P, Cohen V, Small D, Pepe C, Sakr L, Fiset PO, et al: Cytology cell blocks are suitable for immunohistochemical testing for PD-L1 in lung cancer. Ann Oncol 29: 1417-1422, 2018.

This work is licensed under a Creative Commons Attribution-NonCommercial-NoDerivatives 4.0 International (CC BY-NC-ND 4.0) License. 